\title{
Influenza pneumonia: a comparison between seasonal influenza virus and the
} H1N1 pandemic

\author{
R. Riquelme*, A. Torres",, M.L. Rioseco+, S. Ewig ${ }^{\S}$, C. Cillóniz", , M. Riquelme*, \\ C. Inzunza*, E. Polverino ${ }^{\#,}$, Y. Gomez*, M.A. Marcos $^{f}$, C. Contreras*, \\ A. Gabarrús ${ }^{\#, \oplus}$ and R. Fasce ${ }^{\star *}$
}

ABSTRACT: We compared clinical presentation, complications and outcome in patients with influenza $A(H 1 N 1)$ and seasonal influenza pneumonia.

The group of patients with influenza A (H1N1) pneumonia consisted of 75 patients. 52 patients with pneumonia associated with seasonal influenza were included for comparison.

Patients with pneumonia associated with novel H1N1 influenza were younger (mean age $39.7 \mathrm{yrs}$ versus $69.6 \mathrm{yrs}$ ) and had fewer chronic comorbidities and less alcoholism. Infiltrates were more extensive and frequently interstitial. Respiratory failure was more frequent (those with an arterial oxygen tension/inspiratory oxygen fraction ratio $<20028 \%$ versus $12 \%, p=0.042$ ), leading to a higher rate of intensive care unit (ICU) admission and mechanical ventilation $(29.3 \%$ versus $7.7 \%$ $(p<0.0030)$ and $18.7 \%$ versus $2 \%(p<0.0045))$. Mortality was twice as high in patients with novel H1N1 (12\% versus $5.8 \% ; p=0.238$ ), although this was not significant, and was attributable to pneumonia in most instances $(77.8 \%$ versus $0 \% ; p=0.046)$.

Younger age, fewer comorbidities, more extensive radiographic extension and more severe respiratory compromise, and ICU admissions are key features of the clinical presentation of patients with novel H1N1-associated pneumonia compared with seasonal influenza pneumonia.

KEYWORDS: Community-acquired pneumonia, influenza A (H1N1) pneumonia, seasonal influenza pneumonia, viral pneumonia

tudies assessing the aetiology of community-acquired pneumonia have unanimously shown influenza virus to be a frequently involved pathogen. The reported frequencies vary between $4 \%$ and $19 \%$ [1].

Seasonal influenza virus infection has been shown to be associated with considerable excess mortality, particularly in elderly and comorbid patients, at least in seasons with high influenza activity. Recently, from the 1976-1977 to the 2002-2003 seasons, an annual average of $>25,000$ influenzaassociated respiratory and circulatory deaths (9.9 deaths per 100,000) have been calculated in the USA, mostly affecting the young (aged $<5 \mathrm{yrs}$ ) and the elderly (aged $>50 \mathrm{yrs}$ ) [2].

The pandemics in 1918 (H1N1), 1957 (H2N2) and 1968 (H3N2) were characterised by major excess morbidity and mortality as a consequence of viral reassortment. For the same reason, the novel influenza A (H1N1) virus was suspected to be highly pathogenic since its rapid spread in Mexico in 2009. Therefore, the emergence of novel H1N1 influenza in 2009 has been a major challenge for public health and medical institutions and a global pandemic was declared in July 2009 [3].

Early on in this recent pandemic, it became evident that there are consistent differences in host characteristics, clinical presentation and outcomes between patients with novel H1N1 and seasonal influenza virus [3, 4]. We therefore compared these features in large populations presenting with seasonal and novel H1N1 influenza virus-associated pneumonia.

\section{METHODS}

\section{Inclusion criteria and definitions}

The study was approved by local Ethics Commitees (Hospital Clinic (Barcelona, Spain) and Hospital Puerto Montt (Puerto Montt, Chile); reference number 2009/5251). Immunocompetent patients aged $>16 \mathrm{yrs}$ and with confirmed influenza virus infection and community-acquired pneumonia were included in the analysis. Immunocompetence was defined as the absence of
AFFILIATIONS

*Universidad San Sebastian, Servicia de Medicina Interna, Hospital de Puerto Montt,

+Departamento de Microbiología, Hospital de Puerto Montt, Puerto Montt,

**Departamento de Virología, Instituto de Salud Pública de Chile, Santiago, Chile,

\#Servicio de Neumología, Instituto Clínico del Tórax, Hospital Clínic i Provincial de Barcelona, Insitut d'Investigacions Biomèdiques August Pi i Sunyer, Universidad de Barcelona, Ciber de Enfermedades Respiratorias,

${ }^{f}$ Departamento de Microbiología, Hospital Clínic i Provincial de Barcelona, Barcelona, Spain,

"Programa de Investigación en Gripe, ISCIII, Ministerio de Ciencia,

Gobierno de España, and

${ }^{\text {s}}$ Thoraxzentrum Ruhrgebiet, Kliniken für Pneumologie und Infektiologie, Herne und Bochum, Bochum, Germany.

CORRESPONDENCE

A. Torres

Hospital Clínic i Provincial de

Barcelona

Villarroel 170

Esc. 6-8

2a planta

08036 Barcelona

Spain

E-mail: atorres@clinic.ub.es

Received:

Aug 062010

Accepted after revision:

Oct 182010

First published online:

Nov 252010

European Respiratory Journal Print ISSN 0903-1936

Online ISSN 1399-3003 
neutropenia, solid organ or stem cell transplantation, HIV infection and any immunosuppressive treatment, including oral steroid treatment at daily dosages of $>15 \mathrm{mg}$.

Community-acquired pneumonia was diagnosed in the presence of a new infiltrate on chest radiography together with symptoms of lower respiratory tract infection as well as the absence of alternative diagnoses at follow-up. Pneumonia was classified as the reason for hospitalisation in the absence of other evident reasons, e.g. decompensated comorbidity. Bacterial pneumonia cases were excluded. Death was attributed to pneumonia clinically in the absence of other lethal complications.

\section{Patient populations}

Overall, 52 patients with pneumonia associated with seasonal influenza were included in the study. Of these, 42 were consecutively diagnosed and admitted between October 2003 and December 2008 at the Hospital Clinic in Barcelona, Spain. 10 corresponded to patients hospitalised at the Hospital de Puerto Montt between April 2005 and March 2006. Diagnosis of seasonal influenza was established by seroconvertion, i.e. a four-fold increase of immunoglobulin $\mathrm{G}$ in the complement fixation test (Barcelona) or the haemagglutination test (Puerto Montt).

The group of patients with novel H1N1-associated pneumonia consisted of 75 patients admitted during May and July 2009 in Puerto Montt. These patients were diagnosed by PCR.

\section{Microbiological investigations}

Patients with seasonal influenza were part of a prospective aetiological study including blood cultures, sputum samples, antigen testing for Legionella pneumophila serogroup 1 and Streptococcus pneumoniae, as well as serology in both settings. Patients with H1N1were investigated according to the decision of the attending physicians.

\section{Data}

The following parameters were recorded at admission: age, sex, comorbidity, smoking and alcohol habits, previous antibiotic therapy in the past month before the flu episode, symptoms (fever, chills, cough, sputum and chest pain) and clinical findings (crackles). Pneumonia severity was assessed by the Pneumonia Severity Index (PSI) [5] and CRB-65 score (confusion, respiratory rate, blood pressure and age $>65 \mathrm{yrs}$ ) $[6,7]$. CURB-65 score (confusion, urea, respiratory rate, blood pressure and age $>65 \mathrm{yrs}$ ) was not available for all patients (blood urea value) due to the healthcare emergency (H1N1 pandemic), which required rapid decision-making processes in the emergency department for the evaluation of patients. Radiographic patterns were classified as alveolar, interstitial or mixed and the presence of pleural effusion was assessed. Moreover, all patients were assessed at admission and daily during follow-up for the presence of confusion, hypotension (systolic blood pressure $<90 \mathrm{mmHg}$ ), renal failure, bilateral infiltrates, septic shock, intensive care unit (ICU) admission and mechanical ventilation administration.

\section{Statistics}

Categorical variables were described by frequencies and percentages, while continuous variables were described by means and standard deviations or the median and interquartile range for those data not normally distributed (Kolmogorov-Smirnov test). Categorical variables were compared by the Chi-squared test or Fisher's exact test where appropriate. Continuous variables were compared by unpaired t-test once normality was demonstrated; otherwise, the nonparametric MannWhitney U-test was performed.

Univariate and multivariate logistic regression analyses were performed as an explanatory analysis to predict 30-day mortality (dependent variable) in patients with novel H1N1associated pneumonia. The independent variables were: age, sex, pregnancy, duration of symptoms, previous antibiotic therapy, structural lung disease, chronic heart failure, neurological disorder, alcoholism, radiography, temperature, leukocytosis, arterial partial pressure of oxygen $\left(\mathrm{Pa}_{\mathrm{a}} \mathrm{O}_{2}\right) /$ inspiratory oxygen fraction $\left(F \mathrm{I}, \mathrm{O}_{2}\right)$, mechanical ventilation and shock. Variables that showed a significant result univariately $(p<0.1)$ were included in the multivariate logistic regression backward stepwise model. The Hosmer-Lemeshow goodness-of-fit test was performed to assess the overall fit of the model [8].

All tests were two-tailed and significance was set at 5\%. All analyses were performed with SPSS version 16.0 for Windows (SPSS Inc., Chicago, IL, USA).

\section{Ethics}

A. Torres had access to and takes responsibility for the integrity of the data and the accuracy of the data analysis. This study was approved by Ethics Committee of Hospital Clinic and Hospital de Puerto Montt.

\section{RESULTS}

\section{Comparison of patient characteristics}

Out of the 127 patients enrolled in the study, 52 were hospitalised for seasonal influenza (mean \pm SD $69.6 \pm 17.0 \mathrm{yrs}$ ) and 75 for novel H1N1 influenza (mean \pm SD $39.7 \pm 16.7$ yrs; 75 (75\%) out of 100 patients admitted with confirmed H1N1). All patients had radiologically confirmed pneumonia. This was the cause of hospitalisation in $96.2 \%$ versus $77.3 \%(p=0.004)$ for seasonal influenza and novel H1N1 influenza, respectively, whereas the remainders were hospitalised essentially because of unstable comorbidity $(3.8 \%$ versus $17.3 \%$; $p=0.021)$.

The main clinical characteristics are given in table 1. Chronic heart failure, structural lung disease, neurological disorders and alcoholism were more frequent in patients with seasonal influenza and asthma than in those with novel H1N1. The latter presented more frequently with fever and tachycardia. Significant differences also related to the inflammatory response, with more novel $\mathrm{H} 1 \mathrm{~N} 1$ patients having leukocyte counts $<10,000$ cells $\mu \mathrm{L}^{-1}(61.6 \%$ versus $32.7 \% ; \mathrm{p}=0.001)$ or leukopenia $\left(<4,000\right.$ cells $\left.\mu \mathrm{L}^{-1}\right)(13.7 \%$ versus $0 \% ; \mathrm{p}=0.005)$. The most obvious differences were present on chest radiography. Alveolar infiltrates were more frequently present in patients with seasonal influenza ( $94.2 \%$ versus $46.7 \%$; $p<0.0001)$, whereas the pattern was more frequently interstitial and mixed in those with novel H1N1 (53.3\% versus $5.8 \%$; $<<0.0001$ ). Pleural effusion occurred in four patients with seasonal influenza compared with none in novel H1N1 patients $(p=0.026)$.

\section{Comparison of pneumonia severity}

Pneumonia severity at admission as reflected by CRB-65 and PSI score was higher in patients with seasonal influenza. 
TABLE 1 Characteristics of patients with novel H1N1 and seasonal influenza virus

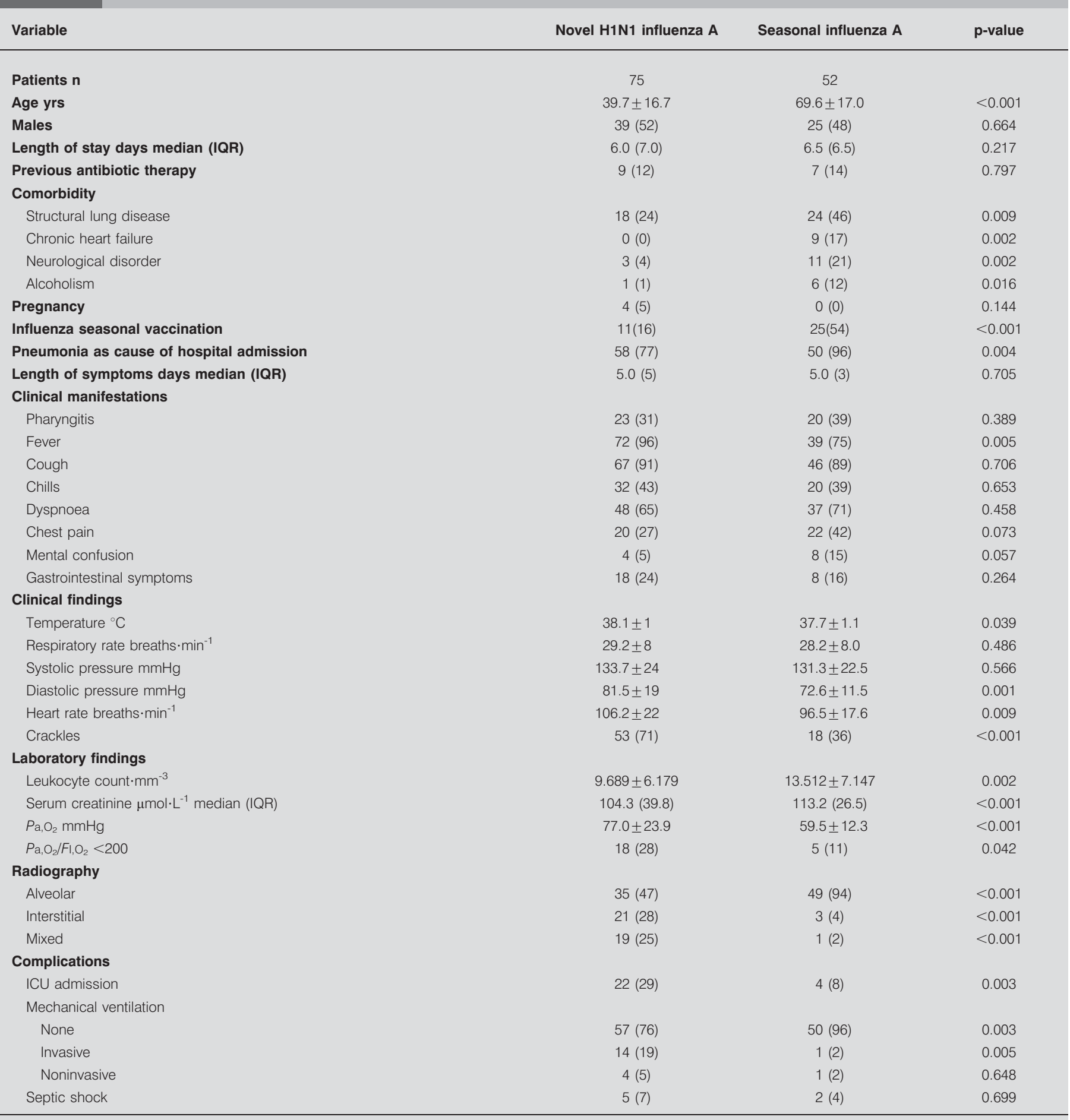

Data are presented as mean $\pm \mathrm{SD}$ or $\mathrm{n}(\%)$, unless otherwise stated. IQR: interquartile range; $\mathrm{Pa}_{1} \mathrm{O}_{2}$ : arterial partial pressure of oxygen; $F \mathrm{I}_{1} \mathrm{O}_{2}$ : inspiratory oxygen fraction; ICU: intensive care unit. $\mathrm{Pa}, \mathrm{O}_{2}$ was on room air.

Both older age and more comorbidities played an important role in determining higher severity scores in the group of seasonal influenza. Overall, $79 \%$ and $60 \%$ of patients with seasonal influenza had scores reflecting higher severity (CRB-65 >1 and PSI class IV-V), respectively, compared with $51 \%$ and $19 \%$ in those with novel H1N1 influenza (table 2).

Low-risk PSI classification was poorly sensitive in patients with novel H1N1, and in those with novel H1N1 and low-risk 


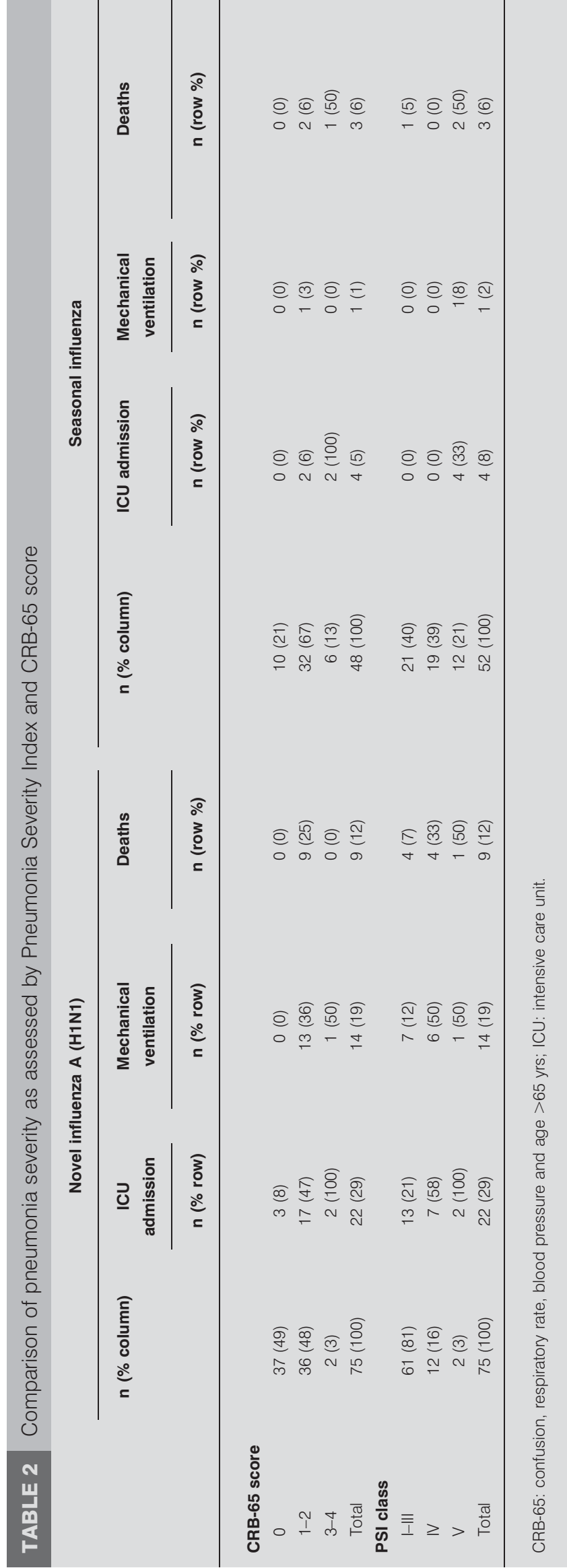

PSI classification (classes I-III), 13 (21\%) out of 61 required ICU admission, seven $(12 \%)$ out of 61 mechanical ventilation, and four $(7 \%)$ out of 61 died. The corresponding numbers for those with seasonal influenza were none for ICU admission and mechanical ventilation and one (5\%) out of 21 for death.

In contrast, low-risk CRB-65 classification (class 0) continued to be associated with a low risk of complications or death. In novel H1N1, three patients required ICU admission, none required mechanical ventilation and there were no deaths. In seasonal influenza, no patients required ICU admission or mechanical ventilation and there were no deaths (table 2).

\section{Microbiological findings}

Blood cultures were retrieved in 32 out of 75 patients with novel H1N1 and 32 out of 52 patients with seasonal influenza. No pathogens were isolated.

Only two cases of seasonal influenza had a co-pathogen (Haemophilus influenzae and respiratory syncytial virus)

\section{Antimicrobial treatment}

All patients received antibacterial treatment according to the recommendations of national guidelines. In patients with novel H1N1-associated pneumonia, this included ceftriaxone monotherapy in 31 patients and ceftriaxone in combination with quinolone in 42 patients; one patient each received macrolide or quinolone monotherapy.

In patients with seasonal influenza, treatment was as follows. In Barcelona, 20 patients received quinolone or $\beta$-lactam monotherapy and 22 patients received combination therapy (11 $\beta$ lactam/quinolone, $10 \beta$-lactam/macrolide and one quinolone/ macrolide). In Puerto Montt, nine patients received ceftriaxone monotherapy and one patient received ceftriaxone combined with a macrolide.

None of the patients with seasonal influenza-associated pneumonia received antiviral treatment. In contrast, all patients except one (74 out of 75) with novel H1N1-associated pneumonia in Puerto Montt received antiviral treatment. Of these, 70 received oral oseltamivir, whereas four pregnant females received inhaled zanamivir. The only patient who did not receive antiviral treatment was diagnosed with some delay but showed a favourable clinical course.

\section{Comparison of complications}

Patients with novel H1N1 were admitted to the ICU far more frequently $(29.3 \%$ versus $7.7 \% ; p=0.003)$. Patients with novel H1N1 influenza had respiratory failure more frequently, as reflected by a $\mathrm{Pa}, \mathrm{O}_{2} / \mathrm{FI}_{1} \mathrm{O}_{2}<200(28.1 \%$ versus $11.6 \% ; \mathrm{p}=0.042)$ and required mechanical ventilation more frequently $(18.7 \%$ versus $2 \%$; $=0.005$ ). Moreover, four patients with novel H1N1 received noninvasive ventilation compared with only one in those with seasonal influenza $(\mathrm{p}=0.648)$. Septic shock was not different between the two groups $(n=5(6.7 \%)$ versus $n=2$ $(3.8 \%) ; p=0.699)$. Length of hospitalisation was not different between groups.

\section{0-day mortality analysis}

30-day mortality was higher in patients with novel H1N1 (12\% versus $5.8 \%$ ); however, this difference did not reach statistical significance $(p=0.238)$. Death was attributable to influenza 
virus in seven $(77.8 \%)$ out of nine patients with novel H1N1 compared with none in those with seasonal influenza $(\mathrm{p}=0.046)$.

Statistically significant variables in the univariate logistic regression analysis and multivariate logistic regression analysis of factors associated with death in patients with novel H1N1-associated pneumonia are presented in table 3. An independent association with death was evident for previous antibiotic therapy, $\mathrm{Pa}_{2} \mathrm{O}_{2} / \mathrm{FI}_{1} \mathrm{O}_{2}<200$, mechanical ventilation and septic shock. Mechanical ventilation was the only variable independently associated with death in multivariate analysis (table 3).

\section{DISCUSSION}

The main findings of the present study are as follows. 1) Pneumonia associated with novel H1N1 influenza affected a different population to seasonal influenza, which was younger (mean 39.7 versus $69.6 \mathrm{yrs}$ ), with far less chronic comorbidity and alcoholism. 2) In clinical terms, these patients had less leukocytosis ( $32.7 \%$ versus $61.6 \%$ ), and more extensive and frequent interstitial infiltrates. 3) Pneumonia presented more frequently with respiratory failure, leading to a higher rate of ICU admission and ventilatory support, particularly mechanical ventilation $(29.3 \%$ versus $7.7 \%$ and $18.7 \%$ versus $2 \%$, respectively). The need for mechanical ventilation was the only independent predictor of death in patients with novel H1N1associated pneumonia. 4) Accordingly, mortality was twice as high in patients with novel H1N1 (12\% versus $5.8 \%)$ and was attributable to pneumonia in most instances (77.8\%). 5) CRB65 , but not PSI, appropriately predicted low-risk patients.

Seasonal influenza epidemics are typically characterised by a J-shaped hospitalisation pattern, with high rates in patients aged $<5$ yrs, low rates in those aged 5-49 yrs and a significant rise in those aged $\geqslant 50$ yrs. Since 1977, H3N2 and H1N1 and influenza B viruses have circulated, with epidemics of H3N2 infection causing the greatest morbidity in the elderly population. Elderly persons $>50$ yrs may be relatively resistant to severe H1N1 disease because of exposure prior to 1957, when these viruses circulated widely. Accordingly, the recent global H1N1 epidemic showed a change in age patterns, with younger persons being frequently infected [3, 4, 9]. In fact,

\begin{tabular}{|c|c|c|c|c|}
\hline TABLE 3 & $\begin{array}{l}\text { nificant univar } \\
\text { ression analys } \\
\text { ients with nov }\end{array}$ & $\begin{array}{l}\text { te and } \\
\text { of } 30-c \\
\text { H1N1 i }\end{array}$ & $\begin{array}{l}\text { ultivariate logis } \\
\text { y mortality for } \\
\text { luenza }\end{array}$ & \\
\hline \multirow[t]{2}{*}{ Variable } & \multicolumn{2}{|c|}{ Univariate } & \multicolumn{2}{|c|}{ Multivariate } \\
\hline & OR $(95 \% \mathrm{Cl})$ & $p$-value & OR (95\% Cl) & $p$-value \\
\hline $\begin{array}{l}\text { Previous antibiotic } \\
\text { treatment }\end{array}$ & $4.9(1.0-24.9)$ & 0.054 & & \\
\hline $\mathrm{Pa}, \mathrm{O}_{2} / \mathrm{Fl}_{1} \mathrm{O}_{2}<200$ & $28.6(3.2-257.6)$ & 0.003 & & \\
\hline $\begin{array}{l}\text { Mechanical } \\
\text { ventilation }\end{array}$ & $40.0(4.5-352.9)$ & 0.001 & $28.0(3.1-251.9)$ & 0.003 \\
\hline Shock & $16.0(2.2-115.3)$ & 0.006 & & \\
\hline
\end{tabular}

$\mathrm{Pa}_{1} \mathrm{O}_{2}$ : arterial partial pressure of oxygen; $\mathrm{Fl}, \mathrm{O}_{2}$ : inspiratory oxygen fraction. younger persons are particularly at risk of severe clinical courses and death. In particular, pregnancy, although not significantly different in our series, was more frequent in patients with $\mathrm{H} 1 \mathrm{~N} 1$ ( $\mathrm{n}=4$ versus $\mathrm{n}=0)$ ).

The classic description of influenza pneumonia was provided by LOURIA et al. [10] after the 1957 H2N2 pandemic. Lower respiratory tract disease was classified into four categories: no radiographic pneumonia, viral infection followed by bacterial pneumonia, rapidly progressive viral pneumonia and concomitant viral-bacterial pneumonia. Mortality was reported to be relatively high. These patterns, however, did not correspond to those typically observed in patients with seasonal influenza-associated pneumonia, particularly in elderly patients $[11,12]$. Prior to significant antigenic shifts, previous exposure to influenza, including vaccinations, may have reduced the severity of influenza-associated lower respiratory tract disease [13].

The clinical presentation of patients with novel H1N1associated pneumonia was different mainly in terms of a higher percentage of patients with pharyngitis, as well as higher mean temperature and heart rate. Although age is a significant risk factor for the development of lower respiratory tract complications of influenza virus infection, pure viral pneumonia is relatively uncommon in immunocompromised hosts outside the pandemic setting. Most elderly persons have partial immunity resulting from vaccination or natural infections [1]. Accordingly, radiographic infiltrates in seasonal influenza have been described as mostly limited, unilateral and subtle [10]. The clinical presentation of our patients with H1N1, however, being characterised by less leukocytosis and more extensive, mostly interstitial radiographic effects, is compatible with pure viral pneumonia of a nonimmune host.

We are unable to analyse the effect of antiviral treatment because virtually all patients with novel H1N1-associated pneumonia were treated with agents active against influenza virus. The higher mortality rate of H1N1-associated pneumonia, despite treatment, compared with seasonal influenza may be due to novel H1N1 itself and does not allow any conclusion. However, some observational data also support the use of antiinfluenza treatment in patients with pneumonia [14].

The mortality rate in our population with novel H1N1associated pneumonia $(12 \%)$ is similar to that reported in previous studies [3,4]. Attributable mortality reached $77.8 \%$. Conversely, patients with seasonal influenza had low mortality $(5.8 \%)$ and none of the deaths of the three patients who died could be attributed to influenza virus infection itself. In multivariate analysis, acute respiratory failure, as reflected by need for mechanical ventilation, was the only independent predictor of death in patients with novel H1N1-associated pneumonia. This finding is compatible with viral pneumonia (and not bacterial co-infection along with septic shock) being the principal cause of death in these patients.

Pneumonia severity assessment tools (PSI and CRB-65) predicted death roughly in a three-class pattern, as expected. However, death rates in higher risk classes were higher than expected in patients with novel H1N1-associated pneumonia and reflected the higher absolute death rates in these patients. Notably, both scores performed equally well in patients with 
seasonal influenza classified as low risk, with only one death in the low-risk PSI group. However, whereas CRB-65 continued to perform well in patients with novel H1N1 pneumonia classified as low-risk classes, high rates of complications and death occurred in the low-risk PSI group. This finding may be explained by the fact that a CRB-65 score of 0 excludes the presence of the main vital sign abnormalities, whereas these may be present in PSI risk classes II and III.

There are several limitations of our study. 1) The population with seasonal influenza was heterogeneous with regards to origin, year and seasonality, which may have biased the comparator; however, our findings fit well with the expected clinical and outcome patterns of these patients. 2) Diagnosis of seasonal influenza was based on paired serology using two different serological methods and may, therefore, have missed cases with acute clinical course and early death; however, other series confirm the relatively low incidence and mortality of pneumonia associated with seasonal influenza [11]. 3) Although the diagnostic approach applied included a search for co-pathogens in patients with seasonal influenza, patients with novel H1N1 were not subject to systematic investigation for co-pathogens and we cannot exactly assess the proportion of patients affected by mixed viral-bacterial aetiologies. However, all patients received antibacterial therapy in accordance with the current international recommendations. The clinical and radiological presentation in novel H1N1 influenza patients suggested pure viral pneumonia in most instances. 4) The international recommendations for the H1N1 pandemic included systematic use of antiviral treatment on hospitalised patients, which was different from the treatment for seasonal influenza. Unfortunately, the effect of differential use of antivirals is difficult to estimate.

In conclusion, the main clinical patterns of pneumonia associated with novel H1N1 influenza differed characteristically from that known for seasonal influenza. Younger age, less comorbidity, more extensive radiographic extension and more severe respiratory compromise are key features. Pregnancy was an additional risk factor. Pneumonia severity was higher, particularly because of acute respiratory compromise, which is also reflected by a higher rate of ICU admission and need for ventilator support. Mortality was double that of seasonal influenza, both in terms of absolute rates and pneumoniarelated mortality. Our data indicate that PSI, but not CRB-65, may underestimate the risk for complications and death in patients classified as low risk.

\section{SUPPORT STATEMENT}

This study has been supported in part by grant number 2009 SGR 911 from Ciber de Enfermedades Respiratorias (Ciberes CB06/06/0028).

Ciberes is an initiative of the Instituto de Salud Carlos III.

\section{STATEMENT OF INTEREST}

Statements of interest for A. Torres and S. Ewig can be found at www. erj.ersjournals.com/site/misc/statements.xhtml

\section{REFERENCES}

1 Falsey AR. Community-acquired viral pneumonia. Clin Geriatr Med 2007; 23: 535-552.

2 Thompson WW, Weintraub E, Dhankhar P, et al. Estimates of US influenza-associated deaths made using four different methods. Influenza Other Respi Viruses 2009; 3: 37-49.

3 Perez-Padilla R, De la Rosa-Zamboni D, Ponce de Leon S, et al. Pneumonia and respiratory failure from swine-origin influenza A (H1N1) in Mexico. N Engl J Med 2009; 361: 680-689.

4 Chowell G, Bertozzi SM, Colchero MA, et al. Severe respiratory disease concurrent with the circulation of H1N1 influenza. N Engl J Med 2009; 361: 674-679.

5 Fine MJ, Auble TE, Yealy DM, et al. A prediction rule to identify low-risk patients with community-acquired pneumonia. $N$ Engl J Med 1997; 336: 243-250.

6 Capelastegui A, Espana PP, Quintana JM, et al. Validation of a predictive rule for the management of community-acquired pneumonia. Eur Respir J 2006; 27: 151-157.

7 Ewig S, Birkner N, Strauss R, et al. New perspectives on community-acquired pneumonia in 388,406 patients. Thorax 2009; 64: 1062-1069.

8 Hosmer DW, Lemeshow S. Applied Logistic Regression. New York, John Wiley \& Sons Inc., 1989.

9 Riquelme R, Riquelme M, Rioseco ML, et al. Characteristics of hospitalised patients with 2009 H1N1 influenza in Chile. Eur Respir J 2010; 36: 864-869.

10 Louria DE, Blumenfeld HL, Ellis JT, et al. Studies on influenza in the pandemics of 1957-1958. II. Pulmonary complications of influenza. J Clin Invest 1959; 2: 135-138.

11 Walsh EE, Cox C, Falsey AR. Clinical features of influenza A virus infection in elderly hospitalized persons. J Am Geriatr Soc 2002; 50: 1498-1503.

12 Falsey AR, Walsh EE. Viral pneumonia in older adults. Clin Infect Dis 2006; 42: 518-524.

13 Jain S, Kamimoto L, Bramley AM, et al. Hospitalized patients with 2009 H1N1 influenza in the United States, April-June 2009. N Engl J Med 2009 12, 361: 1935-1944.

14 Murata Y, Walsh EE, Falsey AR. Pulmonary complications of interpandemic influenza A in hospitalized adults. J Infect Dis 2007; 195: 1029-1037. 